\title{
Complex Interferometry Principles and its Potential in case of Reference Interferograms Availability
}

\author{
Milan Kalal* and Michal Krupka ${ }^{\dagger}$ \\ Faculty of Nuclear Sciences and Physical Engineering, Czech Technical University in Prague, \\ Brehova 7, 11519 Prague 1, Czech Republic \\ kalalefjei.cvut.cz \\ Jan Dostal, Roman Dudzak, and Jiri Ullschmied \\ Institute of Plasma Physics of the Academy of Sciences of the Czech Republic, \\ Za Slovankou 1782/3, 18200 Prague 8, Czech Republic
}

\begin{abstract}
Classical interferometry is one of the key methods in laser-produced plasma diagnostics. Its more advanced version, which allows recording and subsequent reconstruction of up to three sets of data using just one data object - a complex interferogram - was developed in the past and became known as complex interferometry. Employing this diagnostics, not only the usual phase shift, but also the amplitude of the probing beam as well as the fringe contrast (leading directly to the phase shift time derivative) can be reconstructed simultaneously from such a complex interferogram. In this paper it will be demonstrated that even in the case of a not particularly good diagnostic beam quality these three quantities can be reconstructed with a high degree of accuracy provided both the diagnostic beam as well as the corresponding optical line feature a reasonable stability. Such stability requirement is important as all together four shots need to be recorded: the signal complex interferogram, the reference interferogram as well as the intensity structures of the signal and reference part of the diagnostic beam.
\end{abstract}

First EPS Conference on Plasma Diagnostics - 1st ECPD

14-17 April 2015

Villa Mondragone, Frascati (Rome) Italy

\footnotetext{
* Speaker.

${ }^{\dagger}$ Both co-authors are also partially associated with the Institute of Plasma Physics AS CR.
} 


\section{Introduction}

Classical interferometry belongs to the key diagnostics of optically transparent objects (e.g., laser-produced plasmas). By analyzing classical interferograms one can obtain information about the phase shift between the probing and the reference part of the diagnostic beam. There are various ways how to perform such analysis. In this paper we shall concentrate solely on the usage of the fast Fourier transform (FFT), originally proposed for this purpose in its one-dimensional version by Takeda [1] and subsequently extended for two dimensions by Nugent [2], as it paved the way to a more advanced interferogram usability, which allows recording and subsequent reconstruction of up to three sets of independent data from just one data object - a complex interferogram. This technique became known as the complex interferometry (CI) [3],[4]. Using $C I$ approach, not only the usual phase shift but also the amplitude and the fringe contrast (leading directly to the phase shift time derivative) can be recorded simultaneously and subsequently analyzed.

The original idea of $C I$ technique dates back to measurements of magnetic fields spontaneously generated in laser-produced plasmas [5]. This approach provided results with spatial resolutions far superior to those of any similar experiments performed to that time [6]. A more detailed historic overview of circumstances leading to the birth of $C I$ is available [7]. One example of the very first dedicated complex interferogram ever recorded during experiments with generation of spontaneous magnetic fields in laser produced plasma (including its analysis) is provided for illustration (Fig. 1).

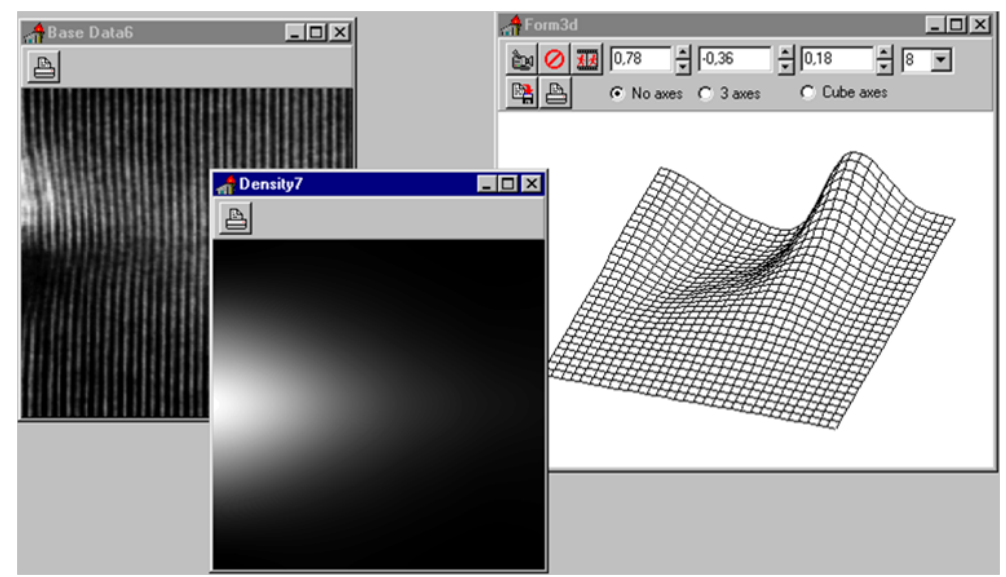

Figure 1: Example of the very first experimentally-obtained complex interferogram (on the left) including reconstructions of the plasma density (in the middle) and the magnetic field (on the right) profiles (M. Kalal and B. Luther-Davies, 1986, ANU, Canberra, Australia). It should be noted that in those early times no reference interferograms were employed.

Over the years this diagnostics has been continuously developed (in particular on the analyzing software side) and successfully employed in recording and analyzing various kind of phenomena. The first major upgrade was the usage of one additional - reference (i.e., signal-free) - interferogram in order to substantially improve the phase shift reconstruction [8]. The second major upgrade dealt with the quality of the amplitude reconstruction utilizing the reference interferogram [9]. However, it became apparent that such an approach would not be sufficient in order to thoroughly overcome the non-homogeneous structure of the diagnostic beam intensity across its cross-section (rather a very practical case). The way how to deal with this inadequacy will be presented in this paper. 


\section{Complex interferometry generalization}

A full mathematical description of $\mathrm{CI}$ basics (without any reference interferograms considered) can be found in [3] and [4]. However, as these particular publications might be somewhat difficult to acquire, one can also look into the easier ones to obtain ([5] and [6]). In particular, the latter of these two contains all the necessary details (but not using the CI terminology, which was introduced only later on).

Our present goal is to perform the third upgrade of the $C I$ theory (the first two were already mentioned in the Introduction) in order to further improve the amplitude reconstruction. The main reason why the second upgrade [9] was nor sufficient lies in the fact that the amplitudes of the signal and reference part of the diagnostic beam were (by default) assumed to be exactly the same (pixel by pixel) on the recording device (without any object influencing the diagnostic beam, of course). This could be, in principle, achieved (after an extremely careful setup) for interferometers with an amplitude division (e.g., Michelson, Mach-Zehnder). In the case of the phase front division (e.g., Nomarski) it is not possible at all. As a result of this fact the formulae (13) and (14) in [9], while correct, are not very practical.

Therefore, a new approach needs to be invented for the purpose of the most precise amplitude reconstructions even in the case of a not very high quality of the diagnostic beam. It will be shown that it can be done. Provided the stability of the diagnostic beam as well as the interferometer setup between the signal and reference shots would be sufficient. On top of that two more reference shots will be required to record intensity structures of the signal and reference part of the diagnostic beam.

Due to this fundamental upgrade it seems useful to perform the derivation of final results from scratch, making it easier to the reader to follow. With only occasional references to already published work. Let us start by the form of an instantaneous intensity $i(y, z, t)$ of an interference field falling in the time $t$ on the point $(y, z)$ of the detector:

$$
\begin{gathered}
i(y, z, t)=a_{s}^{2}(y, z, t) f(t)+a_{r}^{2}(y, z, t) f(t)+ \\
+2 a_{s}(y, z, t) a_{r}(y, z, t) \cos \left[2 \pi\left(\omega_{0} y+v_{0} z\right)+\varphi(y, z, t)\right] f(t) .
\end{gathered}
$$

Here $a_{s}(y, z, t)$ is the amplitude of the signal part of the diagnostic beam; $a_{r}(y, z, t)$ is the amplitude of the reference part of the diagnostic beam (in this $C I$ upgrade it will be assumed that even the reference part can have its own spatial as well as temporal structure); $\varphi(y, z, t)$ represents the phase shift between the signal and reference part of the diagnostic beam; $\omega_{0}$ and $v_{0}$ are the spatial frequencies in $y$ and $z$ direction, respectively. $f(t)$ represents a temporal profile of the diagnostic beam intensity and it is normalized to satisfy the following criteria:

$$
f(t)=f_{s}(t)+f_{a}(t) ; \quad f(t) \geq 0 ; \quad \int_{-\infty}^{+\infty} f(t) d t=\int_{-\infty}^{+\infty} f_{s}(t) d t=1,
$$

where the functions $f_{s}(t)$ and $f_{a}(t)$ represent the symmetric and anti-symmetric part of the function $f(t)$, respectively. Time $t=0$ should be looked for in order to minimize the anti-symmetric part. 
With time $t=0$ properly selected and taking into account that the center of symmetry of $f_{s}(t)$ as well as anti-symmetry of $f_{a}(t)$ will be identical, the following formulae would hold:

$$
\int_{-\infty}^{+\infty} t^{2 n+1} f_{s}(t) d t=0 ; \quad \int_{-\infty}^{+\infty} t^{2 n} f_{a}(t) d t=0 ; \quad n=0,1,2 \ldots
$$

The total superimposed form of the interference field in the $(y, z)$ plane of the detector will be a time integral over the diagnostic beam duration which can be extended to infinity on both sides of the time interval (as outside of the pulse duration $f(t)=0$ ):

$$
i(y, z)=\int_{-\infty}^{+\infty} i(y, z, t) d t
$$

The values $i(y, z)$ represent the structural information of the complex interferogram to be processed. It should be noted, however, that these structures might differ from the recorded ones depending on the response of the detector in use. This is very true, e.g., when a film would be employed as a recording medium (with a subsequent digitization). In case of CCD detectors (and working below their saturation limits) this effect can be usually regarded as insignificant (a simple multiplicative constant having no real influence on the recorded data structure).

From the integral formula (2.4) it becomes obvious that there is no way how to reconstruct the original instantaneous values $a_{s}(y, z, t), a_{r}(y, z, t)$, and $\varphi(y, z, t)$ for every value of $t$. Instead, the Taylor expansion of these quantities in time can be used:

$$
\begin{aligned}
a_{s}(y, z, t) & =a_{s}(y, z)+a_{s}^{\prime}(y, z) t \\
a_{r}(y, z, t) & =a_{r}(y, z)+a_{r}^{\prime}(y, z) t \\
\varphi(y, z, t) & =\varphi(y, z)+\varphi^{\prime}(y, z) t
\end{aligned}
$$

This approach is equivalent to the assumption about the linear change of these quantities during the diagnostic pulse duration $\tau$. This requirement can be always satisfied by taking $\tau$ sufficiently short. Also, for the purpose of the correct phase shift reconstruction, it is important to make sure that the maximal change of the phase shift during the diagnostic pulse duration will not exceed $2 \pi$ (thus less than one fringe):

$$
\varphi_{\max }^{\prime}(y, z) \tau<2 \pi
$$

Taking into account the formulae (2.3) it can be shown that under the above conditions the phase shift changes will play the most significant role and changes of the amplitudes can be neglected.

Considering only these most relevant terms the generalized interferogram formula would be:

$$
\begin{gathered}
i(y, z)=a_{s}^{2}(y, z)+a_{r}^{2}(y, z)+ \\
+2 a_{s}(y, z) a_{r}(y, z)|q(y, z)| \cos \left[2 \pi\left(\omega_{0} y+v_{0} z\right)+\varphi_{\text {total }}(y, z)\right],
\end{gathered}
$$

where

$$
q(y, z)=\int_{-\infty}^{+\infty} f(t) \exp \left[i \varphi^{\prime}(y, z) t\right] d t=\int_{-\infty}^{+\infty} f_{s}(t) \cos \left[\varphi^{\prime}(y, z) t\right] d t+i \int_{-\infty}^{+\infty} f_{a}(t) \sin \left[\varphi^{\prime}(y, z) t\right] d t
$$


and

$$
\varphi_{\text {total }}(y, z)=\varphi_{p}(y, z)+\varphi_{\text {derr }}(y, z)+\varphi_{\text {serr }}(y, z) .
$$

In the expression for the total phase shift (2.9) the meaning of its partial contributions is the following: $\varphi_{p}(y, z)$ stands for the pure phase shift caused by the object itself (this is the quantity we are looking for !!!); $\varphi_{\text {derr }}(y, z)$ stands for the error caused by the diagnostic system itself (interferometer setup and the diagnostic beam wave front quality); $\varphi_{\text {serr }}(y, z)$ stands for the systematic error caused by the degree of asymmetry of the diagnostic pulse $f(t)$.

In the case of the symmetric diagnostic beam profile $f(t)=f_{s}(t)$ the $q(y, z)$ becomes the function with real and positive values only:

$$
q(y, z)=|q(y, z)|=q_{r}(y, z)=\int_{-\infty}^{+\infty} f_{s}(t) \cos \left[\varphi^{\prime}(y, z) t\right] d t ; \quad 0<q(y, z) \leq 1 .
$$

For the more general cases of working with non-symmetric diagnostic beam profiles $f(t)$, where the function $q(y, z)$ will become complex, we also denoted the integral (2.10) as the corresponding real part $q_{r}(y, z)$. For typical symmetric diagnostic beam profiles $f_{s}(t)$ the function $q(y, z)$ is a monotonically decreasing function of $\varphi^{\prime}(y, z)$. This makes finding the inversion process possible, provided the exact time profile of $f_{s}(t)$ is available - either analytically or numerically (by sampling the $f_{s}(t)$ time profile). The reconstructed $\varphi^{\prime}(y, z)$ can be further employed, e.g., for determining the plasma expansion velocity [9].

In the case of the symmetric diagnostic beam profile $f_{s}(t)$ no systematic error $\varphi_{\text {serr }}(y, z)$ will be generated. However, in more practical cases (with some degree of asymmetry of the diagnostic beam profile $f(t)$ ), it would be very useful to have some method for determining the value of this systematic error $\varphi_{\text {serr }}(y, z)$. And, indeed, it can be done. Such a possibility is directly related to the fact that the $\varphi^{\prime}(y, z)$ function can be reconstructed reasonably well even in the case of $f(t)$ asymmetry paving the way to determination of the imaginary part of $q(y, z)$ (i.e., $q_{i}(y, z)$ ) from the integral:

$$
q_{i}(y, z)=\int_{-\infty}^{+\infty} f_{a}(t) \sin \left[\varphi^{\prime}(y, z) t\right] d t .
$$

This approach can be fully justified by taking into account the fact that both anti-symmetric functions $f_{a}(t)$ and $\sin \left[\varphi^{\prime}(y, z) t\right]$ go through zero values at the time $t=0$ (unlike $f_{s}(t)$ and $\cos \left[\varphi^{\prime}(y, z) t\right]$ ), making valid the following inequality:

$$
\left|q_{i}(y, z)\right| \ll q_{r}(y, z) .
$$

Provided the function $|q(y, z)|$ can be reconstructed from complex interferograms, the set of information needed for evaluation of $\varphi_{\text {serr }}(y, z)$ will become complete. Considering (2.12) $|q(y, z)|$ can be regarded as a reasonable approximation for the real part $q_{r}(y, z)$. This will provide the way of reconstructing $\varphi^{\prime}(y, z)$ from the inversion of the integral (2.10). Subsequently, the imaginary part $q_{i}(y, z)$ can be calculated using (2.11). Finally, the $\varphi_{\text {serr }}(y, z)$ itself can be evaluated:

$$
\varphi_{\text {serr }}(y, z)=\arcsin \left(\frac{q_{i}(y, z)}{|q(y, z)|}\right) .
$$




\section{Analysis of complex interferograms employing reference interferograms}

In this Section it will be shown how the complex interferograms can be analyzed using FFT approach and what advantage will be gained from the use of additional sets of reference data (interferograms as well as intensities of the signal and reference part of the diagnostic beam). As this approach is based on the same already published basic principles (e.g., [3]-[6] and [9]) it won't be repeated in all its details. Instead, only the most relevant results will be used in order to start the analysis. It should be emphasized, however, that this time so far the most general method providing the most accurate results will be developed.

In order to obtain information about all three fundamental quantities (i.e., phase shift, amplitude, and phase shift time derivative) through modification of the signal part of the diagnostic beam by the object under observation, two specific quantities available from the Fourier space corresponding to a complex interferogram (2.7) need to be reconstructed using the usual technique: $v(y, z)$ (visibility - from the side lobe) and $b(y, z)$ (background - from the central lobe):

$$
\begin{gathered}
v(y, z)=a_{s}(y, z) a_{r}(y, z)|q(y, z)| \exp \left(i \varphi_{\text {total }}\right), \\
b(y, z)=a_{s}^{2}(y, z)+a_{r}^{2}(y, z) .
\end{gathered}
$$

In the cited literature so far only the individual complex interferograms (already containing some signal) had been considered for processing. Having no corresponding reference interferogram (signal-free) counterpart (with only two exceptions - [8] and [9]). Under such circumstances, however, a reliable reconstruction of the three fundamental quantities was possible only by assuming a very good quality of the diagnostic beam as well as an interferometer setup (optical alignment and quality of its components). Also, the corresponding complex interferograms needed to contain some signal-free parts (for normalization). However, such requirements are, in practice, rarely fulfilled to the full extent.

In the past, there was a good reason for this (limited) approach, though, as in majority of cases the diagnostic beam was derived from the main laser beam (usually with a subsequent conversion to a higher harmonic) to keep the jitter between these two beams at its minimum. Under such circumstances, however, firing a signal-free shot was not regarded as economical. Also, the time delay between two such shots (with the high energy level) would be rather long (tens of minutes), thus not very stable from shot to shot. And using $\mathrm{CW}$ alignment laser as a substitute was far from satisfactory for $C I$ requirements. Only recently, after solving the problem of mutual synchronization, major laser installations (e.g., Prague Asterix Laser System - PALS) started to employ fully independent diagnostic beams (usually Ti:Sa) capable of firing the reference shot with a minimum delay. Which opened the door to manifest a full CI potential.

In order to be able to compensate for typical errors both in the phase shift as well as the amplitude reconstruction, the reference interferograms come very handy. In the generalizations published so far it was assumed (by default) that two interfering parts of the diagnostic beam in the absence of any object would have exactly the same (pixel by pixel) $(y, z)$ structure in the interference/detector plane. This could be, in principle, achieved (after a very careful setup) for interferometers with an amplitude division (e.g., Michelson, Mach-Zehnder). In the case of the phase front division (e.g., Nomarski) it is practically impossible (with the degree of precision required). Total energies between individual shots could vary, but their ratio needs to be taken into account. 
Based on the above analysis it became clear that a new approach should be developed for the most accurate reconstructions even in the case of a not very high quality of the diagnostic beam. The good news is that it can be done. Provided the stability of the diagnostic beam between the reference and signal shots would be sufficient. The same holds for the interferometer setup stability.

Let us denote the signal and reference part of the diagnostic beam in the case of the reference interferogram (signal-free) by the lower index 0. In that case the effect of the object on the amplitude of the signal part of the diagnostic beam generating the complex interferogram can be expressed the following way:

$$
a_{s}(y, z)=f(y, z) a_{s 0}(y, z) \text {, }
$$

where $f(y, z)$ is the multiplicative function containing the amplitude effect information $(f(y, z)=1$ with no effect present). Denoting $s(y, z)$ as the ratio between intensities of the reference $-I_{r 0}(y, z)$ - and the signal - $I_{s 0}(y, z)$ - part of the diagnostic beam for the signal-free shots (two additional separate shots to record these intensities are required and shot-to-shot energy fluctuations need to be taken into account)

$$
s(y, z)=\frac{I_{r 0}(y, z)}{I_{s 0}(y, z)}=\frac{a_{r 0}^{2}(y, z)}{a_{s 0}^{2}(y, z)},
$$

the following set of the most accurate general solutions can be found:

$$
\begin{aligned}
& \varphi_{p}(y, z)=\arctan \left[\frac{\left.\left.\operatorname{Im} \frac{v(y, z)}{v_{0}(y, z)}\right] \operatorname{Re} \frac{v(y, z)}{v_{0}(y, z)}\right]-\varphi_{\text {serr }}(y, z)}{f(y, z)=\sqrt{\frac{1}{p} \frac{b(y, z)}{b_{0}(y, z)}[s(y, z)+1]-s(y, z)}}\right. \\
& |q(y, z)|=\frac{\frac{1}{p}\left|\frac{v(y, z)}{v_{0}(y, z)}\right|}{\sqrt{\frac{1}{p} \frac{b(y, z)}{b_{0}(y, z)}[s(y, z)+1]-s(y, z)}}
\end{aligned}
$$

where $v_{0}(y, z)$ and $b_{0}(y, z)$ are the corresponding visibility and background quantities reconstructed from the reference interferogram and $p$ is the ratio between energies of the diagnostic pulses used for recording of the signal and reference interferograms.

The $\varphi_{p}(y, z)$ is the pure phase shift, free of any errors due to the alignment of the interferometer as well as imperfections in shifting of the side lobe to the center of the Fourier space (as no such shifting is necessary). Also the systematic error $\varphi_{\text {serr }}(y, z)$ caused by the diagnostic beam asymmetric profile $f(t)$ was corrected using (2.13) as well as the imperfections of the phase front structure of the diagnostic beam (as they are the same for the signal and the reference shots).

The $f(y, z)$ function is a pure effect on the amplitude of the probing beam from the object under observation (regardless of the quality of the diagnostic beam). For even better practical results in the amplitude reconstruction the hanning function should be applied to both interferograms.

And eventually the $|q(y, z)|$ function is evaluated (its usage was discussed in detail in the previous Section). 


\section{Concluding remarks}

In this paper so far the most advanced version of the complex interferometry theoretical analysis was presented. It was demonstrated that even in the case of a not particularly good diagnostic beam quality the three fundamental quantities related to the three degrees of freedom (characteristic for $C I$ ) can be reconstructed with a high degree of accuracy provided both the diagnostic beam as well as the corresponding optical line feature a reasonable shot to shot stability. Such stability requirement is important as all together four shots need to be recorded: the signal complex interferogram, the reference interferogram as well as the intensity structures of the signal and reference part of the diagnostic beam. These additional intensity structures (to be recorded separately) are necessary due to the fact that their required ratio cannot be determined unambiguously from the reference interferogram only. Two solutions with mutually inverted values can be found (not included in this paper). But due to the symmetry there is no way how to decide which contribution comes from the signal and which from the reference part of the diagnostic beam.

\section{Acknowledgment}

This work was supported in part by the Ministry of Education, Youth and Sports of the Czech Republic, projects LD14089 and LM2010014, as well as by the Grant Agency of the Czech Republic, project P205-11-P712.

\section{References}

[1] M. Takeda, H. Ina, and S. Kobayashi, Fourier-Transform Method of Fringe-Pattern Analysis for Computer-Based Topography and Interferometry, Journal of Optical Society of America 72 (1982) 156.

[2] K.A. Nugent, Interferogram Analysis Using an Accurate Fully Automatic Algorithm, Applied Optics 24 (1985) 3101.

[3] M. Kalal, Complex interferometry: its principles and applications to fully automated on-line diagnostics, Czechoslovak Journal of Physics 41 (1991) 743.

[4] M. Kalal, Principles of Complex Interferometry, in Optoelectronics for Environmental Sciences, Plenum Press, New York 1991.

[5] M. Kalal, K.A. Nugent, and B. Luther-Davies, Phase-amplitude imaging: its application to fully automated analysis of magnetic field measurements in laser-produced plasmas, Applied Optics $\mathbf{2 6}$ (1987) 1674.

[6] M. Kalal, K.A. Nugent, and B. Luther-Davies, Phase-amplitude imaging: The fully automated analysis of megagaus magnetic field measurements in laser-produced plasmas, Journal of Applied Physics $\mathbf{6 4}$ (1988) 3845.

[7] M. Kalal, Complex Interferometry, in Modern Topics in Physics, ARSCI, Praha 2003 (the relevant chapter available upon request by email).

[8] M. Kalal, O. Slezak, M. Martinkova, and Y.J. Rhee, Compact Design of a Nomarski Interferometer and Its Application in the Diagnostics of Coulomb Explosions of Deuterium Clusters, Journal of the Korean Physical Society 56 (2010) 287.

[9] M. Kalal, Complex Interferometry: how far can you go?, Physics Procedia 62 (2015) 92. 